\title{
Importancia del órgano de solución de diferencias en la OMC en los mercados internacionales
}

\section{Importance of the dispute settlement body in the WTO in international markets}

Tatiana Stefanía Macías Muentes

Kathiana Marilú León Briones

Universidad de Especialidades Espíritu Santo, Ecuador

Autor para correspondencia: tmacias@uees.edu.ec, kmleon@uees.edu.ec.

Fecha de recepción: 12 de diciembre de 2016 - Fecha de aceptación: 10 de Enero de 2017

\section{Resumen}

El presente trabajo tiene por objetivo determinar la importancia del Órgano de Solución de Diferencias de la OMC dentro de las negociaciones sobre el acceso a los mercados internacionales. El área que se está investigando es el funcionamiento de la OMC especialmente, cómo esta resuelve las diferencias entre países, especialmente teniendo en cuenta el análisis de costobeneficio que se incurre. La investigación se lleva a cabo a través de la revisión de fuentes primarias y secundarias con un enfoque cualitativo y comparativo así como perspectiva analítica y descriptiva. El resultado que se obtuvo después de estudiar dos casos específicamente en Ecuador, nos muestra cómo el organismo proporciona buenas oportunidades de desarrollo comercial a nivel mundial con ahorro de recursos. Se recomienda que los países hagan conciencia y permitan que estos organismos les asesoren al momento de tomar decisiones y resolver conflictos en búsqueda de un comercio más eficaz y eficiente.

Palabras claves: Ecuador; Organización Mundial del Comercio; comercio internacional; órgano de diferencias; negociaciones

\begin{abstract}
The purpose of this paper is to determine the importance of the WTO Dispute Settlement Body in the negotiations on international market access. The area under investigation is the functioning of the WTO, especially how it resolves the differences between countries, especially taking into account the cost-benefit analysis that is incurred. The research is carried out through the revision of primary and secondary sources with a qualitative and comparative approach as well as an analytical and descriptive perspective. The result obtained after studying two cases specifically in Ecuador, shows us how the organism provides good opportunities for commercial development worldwide with resource savings. It is recommended that countries raise awareness and allow these agencies to advise them when making decisions and resolve conflicts in search of a more efficient and effective trade.
\end{abstract}

Key words: Ecuador; WTO; international trade and dispute settlement body; negotiation 


\section{Introducción}

En el mundo de hoy, gran porcentaje de la población está arraigada a las distintas dinámicas que el comercio les ofrece, tanto internacional como localmente (Grasstek, 2013, pág. 21). En las últimas décadas, el comercio se ha ido desarrollando a un ritmo no antes visto, gracias a la sinergia que ha surgido con la tecnología y la globalización. Con la ayuda de estas tendencias el comercio ha tenido mayor alcance y aceptación en términos generales. Un claro ejemplo es el siguiente: hipotéticamente un comerciante en Estados Unidos, a quien llamaremos "John" vende celulares a "Pablo" en Ecuador y viceversa, de modo que manteniendo una relación ganar-ganar ambos tratan de abrir sus mercados a mejores oportunidades tomando en cuenta los intereses de cada uno y negociando sus condiciones.

Con este ejemplo se refleja que así como el comercio no solamente existe entre personas, existen dichos individuos que pertenecen a una nación y por esta naturaleza actúan, de manera automática, en representación de la misma. Es así que este simple intercambio también implica el intercambio entre naciones o países. Se desearía que las actividades comerciales sucedieran de manera espontánea y pacífica, sin embargo en algunos casos por, lógicamente, perseguir y defender los intereses de una nación, surgen conflictos. Por ellos, para que pueda existir una manera más organizada y eficaz de tratar el comercio entre países, existe la Organización Mundial del Comercio (OMC).

La OMC es uno de los organismos de mayor relevancia a nivel mundial ya que pretende ayudar a establecer acuerdos legales mundiales que velen por el bienestar y la armonía comercial entre cada país. Esta organización actualmente se conforma de 164 miembros que participan en el comercio mundial y 20 países observadores, los cuales no tienen voz ni voto pero pueden formar parte de las negociaciones, basándose en los acuerdos firmados por estos países miembros.

Aunque la OMC está conformada por varios componentes, este artículo académico se enfocará en el mecanismo de solución de diferencias. Además se analizara la forma mediante la cual, la organización debería gestionar los diferentes conflictos y por consecuencia, penas en el comercio internacional. A pesar de que algunos de los gobiernos no están de acuerdo con las obligaciones de la OMC y piensan que pueden omitir estas reglas con impunidad, todas estas normas se han creado bajo consenso y al ser miembros están intrínsecamente obligados a honrarlas y se deben seguir sin excepción.

Por dicha relevancia del Órgano de Solución de Diferencias de la OMC con respecto a las negociaciones con los mercados internacionales surge la oportunidad de exponer la necesidad que tienen los países de incorporar las normas o reglas que la OMC pone a su disposición, tomando en cuenta los múltiples efectos que podrían darse en la toma de malas decisiones de los diferentes gobiernos. Sin embargo, surgen algunas preguntas a partir de lo antes establecido, las mismas que se pretenderán responder a lo largo de este artículo. Por ejemplo, ¿Conocen todos los países la importancia del Órgano de Solución de Diferencias de la OMC? ¿Qué pasaría si los países no se rigen bajo las decisiones ofrecidas por la OMC?, ¿En qué afecta a los mercados de un país con respecto a otro? 
La respuesta se dará desde una perspectiva analítica y descriptiva dando a conocer el poder de decisión que tiene esta organización al momento de intentar penetrar a mercados extranjeros. El estudio se llevará a cabo a través de la revisión de fuentes primarias esencialmente, y secundarias de ser necesario, con un enfoque tanto cualitativo como comparativo ya que tomaremos en cuenta disputas anteriores entre países. Este análisis tendrá como fin determinar aspectos positivos y negativos de los distintos casos a revisar, y se denotarán conclusiones y recomendaciones que sean pertinentes.

\section{Revisión de la Literatura}

\section{Origen de la OMC}

Las economías nacionales están íntimamente relacionadas con el comercio internacional. Esta relación se la puede percibir más como consumidor, ya que al momento de comprar algún producto, la mayoría son fabricados en mercados internacionales. Por ejemplo, la empresa Dell cuenta con una presencia global de 11 fábricas alrededor del mundo, una puede ser ensamblada en Brasil pero sus componentes tal vez fueron diseñados en China, India o Malasia (DELL Company, 2007). Otro claro ejemplo es Apple, una de las empresas más conocidas a nivel mundial, por la gama de productos e innovación que posee. Apple diseña todos sus productos en California pero fabrica y ensambla en China ya que si se lo fabricara en Estados Unidos sus costes resultarían más elevados y han optado por, no solamente internacionalizar la manufactura sino que también la producción de bienes y servicios (Apple, 2012). Sin embargo, gracias a los distintos intereses que cada comerciante persigue, y esta dinamización del mercado que se produce por iniciativas individuales de carácter espontáneo es que, el crecimiento del comercio se puede ver reflejado en los mercados globales y en las distintas normas establecidas por la OMC.

Este organismo nace el 1 de enero de 1995, y es una de las mayores reformas al comercio internacional desde la Segunda Guerra Mundial. Inicialmente se consagró con el nombre de GATT ${ }^{1} \mathrm{y}$ antes de este nombre tuvo un intento fallido de ser concebido como Organización Internacional de Comercio (OIC). Siendo GATT tuvo un sistema ordenado y fructífero mediante rondas de negociaciones comerciales, sin embargo en el año 1980, el sistema necesitaba una reestructuración lo cual condujo a la Ronda de Uruguay que dio lugar a la creación de la OMC (Grossman, 2010, pág. 138).

En el año 2004, la OMC aprobó las Normas de Procedimiento del Parlamento y en 2008 se llevó a cabo la primera etapa de institucionalización de la misma. Pero hay una regla que establece a los parlamentarios deben reunirse al menos una vez al año y ellos no tuvieron ninguna reunión durante 2007, 2009 y 2010, lo que significa que la OMC se hizo más débil. Sin embargo, en 2011, en la Conferencia de Ginebra en la cual conceptualizan la dimensión parlamentaria en un campo de múltiples niveles, pero si nos referimos a un campo de múltiples niveles, es demasiado general y para una mejor comunicación y transparencia entre los países deben ser más específico (Grossman, 2010, pág. 142).

\footnotetext{
${ }^{1}$ GATT por sus siglas en ingles se conoce al: "General Agreement on Tariffs and Trade".
} 
Es relevante tomar datos históricos como referencia que sirva de punto de partida para conocer con mayor detenimiento cómo era la estructura de la OMC y su sistema político. Esta organización nace en los años 1986-1994 siendo resultado de las negociaciones mantenidas en la Ronda Uruguay teniendo como marco teórico anteriormente el GATT y obligan a los gobiernos de los países a garantizar su sistema político mediante la notificación de sus políticas comerciales a la OMC (Organización Mundial del Comercio, 2016). Estas instituciones desempeñan un papel importante no solo para la generación presente sino, también para las futuras generaciones, que son quienes tienen en sus manos el bienestar del país optando por mejores decisiones, teniendo siempre en cuenta las posibles consecuencias que podrían presentarse. Otro aspecto importante es la legitimidad, la OMC debería tener la autoridad y validez en toda regla o castigo que se aplican, ya que se necesitan para equilibrar los diferentes intereses y necesidades de cada país, es cierto que no se puede establecer normas que complazcan a todos los países, pero lo que trata esta organización es que exista un balance en todas las situaciones que tienen que hacer frente y nadie salga afectado a causa de terceros.

\section{Ecuador y la OMC}

El mundo y la globalización han sido factores clave que han contribuido en la evolución alcanzada con respecto a la relación entre Estados. El pasado GATT y la actual OMC, han contribuido a dispersar las fronteras estatales y reducir la posibilidad de que estas organizaciones tienen el control de sus gobiernos. Estas normas comerciales internacionales han sido diseñadas para facilitar las diferentes normas y reglas llevando a cabo transacciones comerciales relacionadas con los exportadores e importadores de bienes y servicios mediante la mejora del bienestar de algunos países (Bouzas \& Ffrench-Davis, 2000).

La Organización Mundial de Comercio desempeña un papel fundamental en las relaciones comerciales entre las naciones. Se ofrece muchas alternativas para las negociaciones con un propósito específico, el cual es aplicar cualquier tipo de restricción en el comercio internacional ayudando al desarrollo del crecimiento económico y político de cada país por ejemplo: reducir los aranceles del camarón para que permita a los países en desarrollo mejorar sus importaciones (Organización Mundial del Comercio, 2016). Teniendo en cuenta que al reducir esos aranceles también pueden afectar indirectamente a todas las personas que viven dentro de una misma nación.

Ecuador, nación que ha formado parte de la OMC desde 1995, y que participó como uno de los 21 países que sin haber formado parte de la Ronda Uruguay, fue el primero que decidió negociar su ingreso a la OMC sin ser parte del GATT (El Comercio, 2015). Inició su proceso dentro de los parámetros que habían sido aplicados para más de cien países adheridos al Acuerdo General. Sin embargo, durante el proceso se ajustó nuevas normas que estaban en vigencia para lograr ser parte de la OMC, siendo así parte de una lista de más de veinte países como China, Rusia o Arabia Saudita que buscaban ser parte de un nuevo sistema multilateral del comercio. Un elemento favorable para Ecuador, es que registraba una importante concentración en lo que respecta a mercados de exportación, Estados Unidos fluctuaba entre el $38 \%$ y el $42 \%$ mientras que la Unión Europea registró el 17\% como mercados de destino de exportaciones ecuatorianas (Ramírez, 2002; Cristian, 2003). Sin embargo, uno de los principales objetivos de la OMC es que exista comercio sin discriminación en el mundo, por ende, los países miembros tienen la 
obligación de cumplir las normas, en el caso de Ecuador ha respetado y resuelto sus distintos casos de diferencias en los cuales como reclamante y demandado ha tenido 3 casos y como tercero 32 casos (OMC y Relaciones Exteriores, 2015).

\section{Sistema de solución de diferencias}

El proceso de solución de diferencia dentro de la OMC es la piedra angular en los convenios multilaterales ya que brinda la estabilidad de una economía mundial a todos los países miembros. Este sistema está basado en normas y reglas que deben ser honradas por los países, puesto que sin este organismo la relación comercial sería menos eficaz. Dentro de las normas que se establecen, también se establecen plazos para ultimar el procedimiento adoptado por un grupo especial miembros de la organización, el cual es el encargado de aprobar o negarlas. Cabe mencionar además que existe la posibilidad de apelar, en cuestiones de Derecho.

Se debe enfatizar que una de las prioridades de la OMC es resolver las diferencias, más no dictar sentencia, la mayoría de veces mediante la celebración de consultas. En Enero de 2008 solo en 136 de los 369 casos planteados se había llegado al final del procedimiento, y el resto la mayoría de los casos habían notificado una prolongación, que al final quedó inconclusa (OMC y Relaciones Exteriores, pág. 57). El mecanismo de este sistema era considerado como ineficaz, consumista e innecesario ya que se perdía tiempo y recursos sin dar por hecho el término de las distintas disputas.

Esta organización recurre al órgano de diferencias cuando se estima que otros miembros infringen las normas comerciales, ya sea entre países desarrollados o en desarrollo. Al momento de infringir las normas comerciales, los países que son parte de la organización deben respetar los procedimientos convenidos y las normas que ya han sido establecidas. Uno de los problemas más comunes frente a una diferencia surge en el momento en el cual un país adopta una medida que otro u otros miembros de la OMC consideran como infracción, originando conflictos de intereses sobre las normas establecidas y es en ese momento la OMC actúa, siendo intermediario entre ambas partes.

En el GATT, ya existía un proceso de solución de diferencias pero no con plazos fijos, es por eso que en muchos de los casos pasaba mucho tiempo sin llegar a una respuesta definitiva, por ejemplo en el caso "Modificación de la Ley de protección de Calidad del Aire, de los Estados Unidos" tuvo una duración de 5 años desde 1990, que fue la iniciación del procedimiento y por motivos de una mala administración, las disputas se demoraban muchos años en llegar a un acuerdo (Organización Mundial del Comercio, 2016). Sin embargo, en el acuerdo de la Ronda Uruguay se desarrollaron actividades más estructuradas de acuerdo al tiempo que debería tomarse en resolver una diferencia, con plazos flexibles para las diversas etapas del proceso (Organización Mundial del Comercio, 2016).

Como procedimiento regular se inicia con una solicitud de celebración de consultas, en el que el Gobierno del país presenta el caso y expone sus objeciones, ambas partes están obligadas a mantener consultas durante 60 días con la finalidad de negociar una solución que beneficie a ambos. Según las estadísticas se logra resolver el $46 \%$ de las diferencias presentadas y solo tres cuartas partes de ellas dan lugar a concesiones. La evidencia refleja que la mayoría de las 
diferencias planteadas se dan entre los países desarrollados como Alemania y EEUU mientras que los países considerados en desarrollo por otro lado, tienen poca experiencia en solución de diferencias; China participa cada vez más activamente en solución de diferencias en calidad de terceros, tratando de obtener experiencia con el sistema (Busch \& Reinhardt, 2004).

\section{Cómo resolver las diferencias entre países}

El Órgano de Solución de Diferencias se ha ido desarrollando de manera más eficiente estableciendo nuevas normas y plazos para vigilar la aplicación de las resoluciones teniendo la potestad de autorizar nuevos cambios con el fin de mejorar el proceso. Este Órgano consta de algunas etapas, en el gráfico No.1 podemos observar cuáles son y a continuación se detallarán en qué consiste cada etapa con sus respectivos plazos.

Para resolver una diferencia, se desarrolla a través de tres etapas principales: consulta, etapa contenciosa formal y si es necesario aplicación. Todo caso empieza en la etapa de consultas y, su plazo máximo es de 60 días. Es por ello, que antes de adoptar cualquier medida, los países involucrados deben reunirse para dialogar con el propósito de tratar de resolverlo por su cuenta. Una vez agotado este recurso de manera no fructífera, se puede solicitar al Director General de la OMC que actúe como mediador, entre los países involucrados con el fin de llegar a un acuerdo mutuo que beneficie a ambas partes (OMC y Relaciones Exteriores, pág. 58).

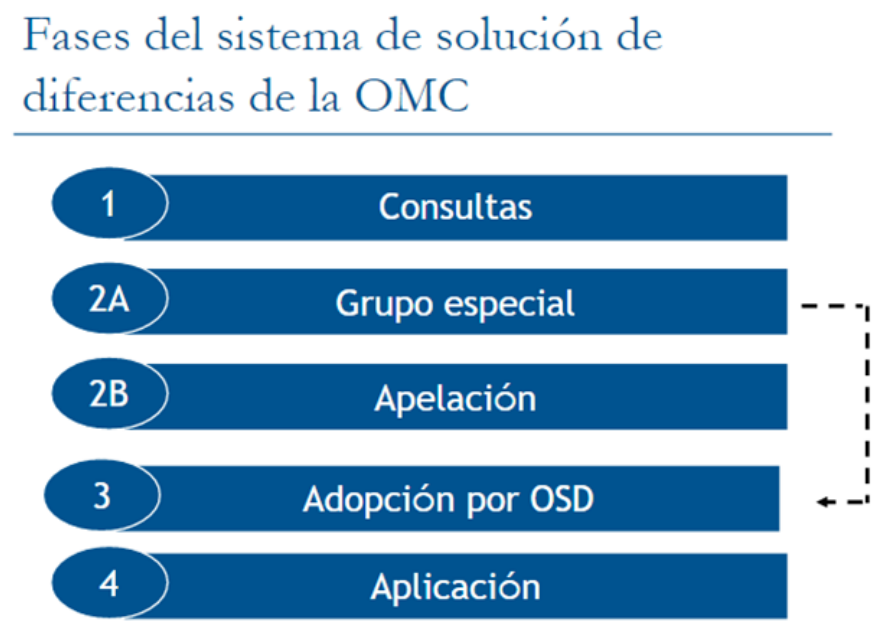

Grafico 1: Fases del Sistema de Solución de Diferencias de la OMC Fuente: (OIC, 2016)

En la segunda etapa, una vez hecho el intento de la consulta entre los países, y no habiendo llegado a una solución satisfactoria, el país reclamante puede pedir que se establezca un grupo especial. El grupo especial es aquel que, en un plazo no mayor a 45 días, ayuda al Órgano de Solución de Diferencias a tomar medidas acerca del caso en cuestión. Por último, si no se pudo llegar a un acuerdo en la segunda etapa, la tercera es la aplicación de la resolución. En este momento del proceso, se sugiere recurrir a medidas más drásticas con la parte demandante ya que se asume que no estaría colaborando en el cumplimiento de acuerdo y se daría por terminado el caso (OMC y Relaciones Exteriores, pág. 59). 
La situación de la OMC es cada vez más preocupante debido a que algunos países como México, Hong Kong y China están en contra de las diferentes normas que la OMC tiene para el libre comercio, anotando una victoria importante para los países más poderosos y más ricos. Si ellos no tienen apoyo, su sistema de solución de diferencias se disminuye aún más y, deben encontrar la manera de velar por el bienestar común en el comercio internacional y cambiar la ideología de estar a favor del país más rico y tratar de ser más neutral en el proceso de toma de decisiones de las políticas y de las penalidades. Este tipo de mecanismo es muy útil y productivo, pero solo si se sabe analizar las medidas necesarias con respecto a los diferentes niveles económicos y político que existe entre países. (Bello, 2006).

\section{Costo- Beneficio sobre el órgano de diferencias}

Uno de los factores importantes que se deben tomar en cuenta al momento de presentar una solución de diferencias es el costo-beneficio. Analizar el posible costo que implica dicho proceso, que por lo general dura un año en concluir, más un año de implementación y un poco menos de un año en caso de surgir desacuerdos entre las propuestas que propone cada país. No solo es el costo- beneficio en la parte económica sino también con respecto al tiempo. Ambas partes deben poner en una balanza los posibles costos económicos que obtendrían en caso de litigar y por otra parte, el costo incurrido. La inversión aproximada asciende a los cientos de miles de dólares y en algunos casos millones de dólares. Por esta razón los países involucrados deben ser asesorados por abogados externos ${ }^{2}$, los cuales pueden ser privados o del Centro de Asesoría Legal de la OMC para que de acuerdo a su caso puedan tomar la mejor decisión (CEPAL , 2008).

Por otra parte, existen otras alternativas para presentar reclamos ante la OMC y pueden resultar menos costosas como por ejemplo: los buenos oficios, la conciliación y la mediación. Son procesos de forma voluntaria en los que se, requiere que ambas partes estén de acuerdo. Por la naturaleza de estos procesos, se puede recurrir a ellos en cualquier momento y debe existir confidencialidad; la OMC ofrece estos servicios algunas veces de forma voluntaria sin la necesidad de una solicitud previa.

Otro mecanismo alternativo y el más utilizado es el Arbitraje. Este mecanismo al igual a los previamente mencionados; de tipo voluntario y requiere el acuerdo entre las partes sobre las reglas de procedimiento. Una de las ventajas principales del arbitraje es que las partes tienen la libertad de establecer reglas o normas ajustadas a sus propios intereses sin el requisito que un tercero que intervenga.

Además, existen alianzas estratégicas en las cuales el país reclamante puede solicitar una alianza con otro Miembro de la OMC para que la presión con respecto al otro país sea más fuerte, también puede apelar a la ayuda de organizaciones no gubernamentales, a nivel internacional o regional siempre y cuando las medidas no afecten los intereses sociales entre países, como el medio ambiente o la salud humana (CEPAL, 2008).

\footnotetext{
${ }^{2}$ Los abogados externos deben tener algunas características específicas, debe ser un profesional independiente que no tenga relación alguna con el caso en cuestión y que preste sus servicios a los clientes que libremente lo contraten. El pago a estos abogados se realiza por una remuneración periódica dependiendo sea el caso. Algunos ejemplos de abogados: CONSULEGIS S.A., SUAREZ Consultoría.
} 


\section{Metodología}

El enfoque metodológico de este estudio es cualitativo. Se pretende comparar casos específicamente de Ecuador en los años 2002-2010, que estén relacionados con las diferentes disputas que se hayan presentado, analizar las perspectivas y diferentes puntos de vista con que la OMC aplicó normas, resoluciones o sanciones. También se pretende analizar por qué los países desarrollados son los que presentan un mayor número de casos de diferencias a comparación de los países en desarrollo así como reflejar cuáles son las posibles implicaciones de no tomar en cuenta las normas establecidas por la OMC en mercados internacionales. Más adelante, se analizarán las posibles conclusiones y recomendaciones para poder fortalecer el poder que debería tener la OMC frente a los gobiernos de los demás países cumpliendo con el principal objetivo de garantizar que el comercio internacional sea equitativo y sostenible para el crecimiento económico y desarrollo mundial.

Tabla1: Diferencia en que participa o ha participado Ecuador.

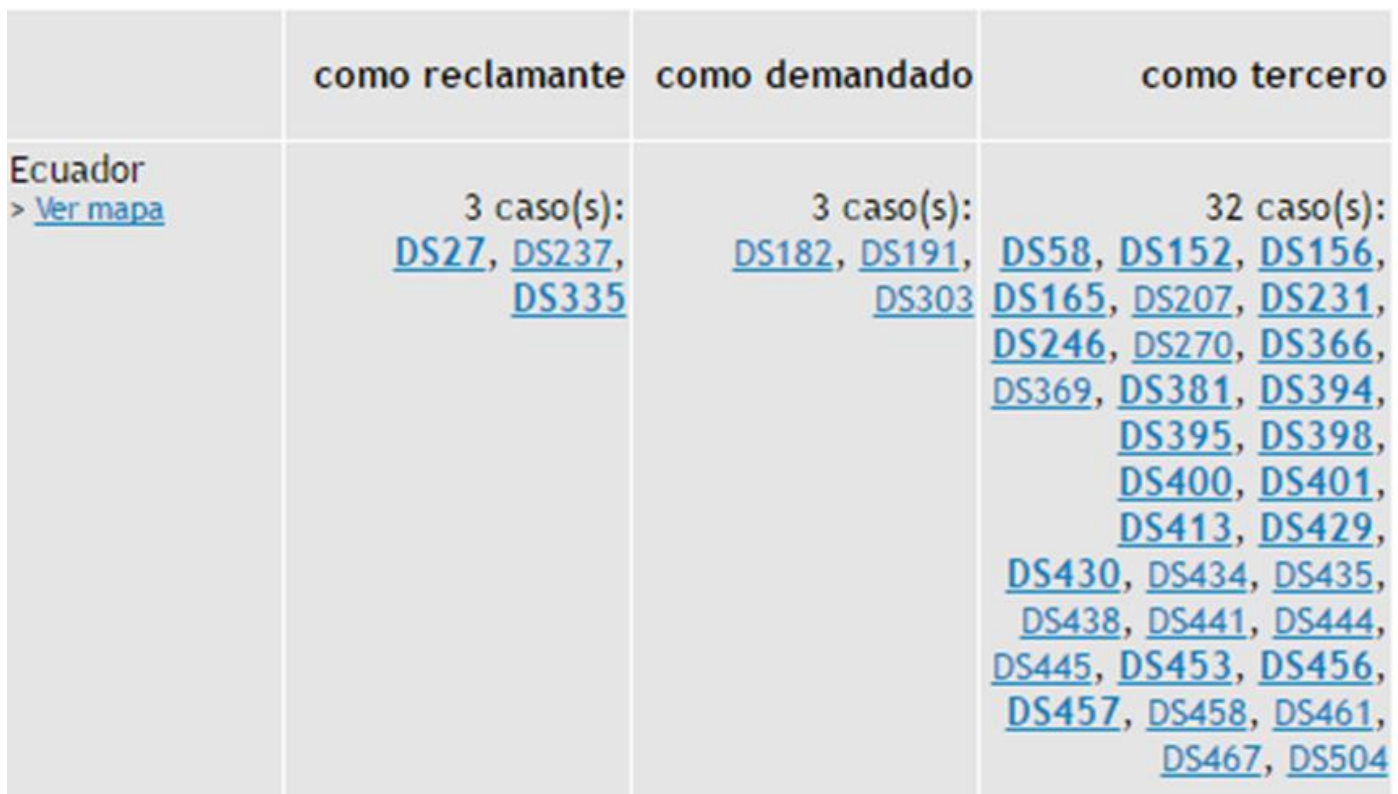

Fuente: (Organización Mundial del Comercio, 2016)

\section{Análisis de los Resultados}

\section{Antidumping (EEUU - Ecuador)}

Uno de los casos más relevantes en los que Ecuador ha solicitado la intervención de la OMC es el del dumping del camarón, teniendo como demandado a Estados Unidos. La disputa consiste en el alza de impuesto que Estados Unidos le aplica al camarón ecuatoriano en el mercado al darse cuenta que en otros mercados se está vendiendo el mismo producto a un menor precio del que ellos lo comercializan, lo cual trajo como consecuencia una competencia desleal. 
La disputa empieza en el 2004, cuando Estados Unidos realizó una investigación sobre los precios en los que se comercializaba el camarón en los mercados norteamericanos, en la cual Ecuador, Brasil, India y Tailandia estaban exportando camarones a precios inferiores del costo real. Por esta razón EEUU decidió imponer un arancel a sus importaciones ya que el margen de dumping para Ecuador era de hasta el 4.48\% (ICTSD, 2007).

A partir de este acontecimiento, el 17 de noviembre de 2005, Ecuador solicitó a la OMC que: (1) Estados Unidos redujera el precio al producto tomando en cuenta la intervención del Departamento de Comercio de los Estados Unidos que propuso la revocación los aranceles antidumping del camarón procedente del Ecuador, y (2) que se permita el ingreso del camarón sin aranceles al mercado estadounidense. Tomando en cuenta los intereses de ambos países en materia de exportaciones, la OMC permitió el fallo favorable al Ecuador por el caso "Estados Unidos y medidas antidumping”, dándole un plazo prudencial no mayor a 6 meses para que puedan establecer un procedimiento de normas antidumping según la OMC (Díaz \& Regalado Eduardo, 2001).

La presencia del sistema de diferencias de la OMC desarrolla un papel primordial al momento de tomar decisiones, en el caso de Estados Unidos, es uno de los principales socios comerciales para Ecuador como se puede observar en el gráfico 1, dentro de la inversión extranjera Estados Unidos es el segundo país que invierte directamente a Ecuador con el 16.6\%, siendo Holanda el primero en la lista (79.3\%). En el momento que decidió Estados Unidos subir aranceles sin previo aviso, no solo iba a afectar a Ecuador sino a la economía mundial. Tomando en cuenta que Estados Unidos es uno de sus principales socios exportadores, a ambos les conviene tener una muy buena relación bilateral y poner fin a este caso de diferencias para que a futuro puedan negociar mejores oportunidades. El caso concluye cuando la OMC le otorga un plazo a Estados Unidos para reivindicarse y establecer un arancel acorde a lo establecido con Ecuador.

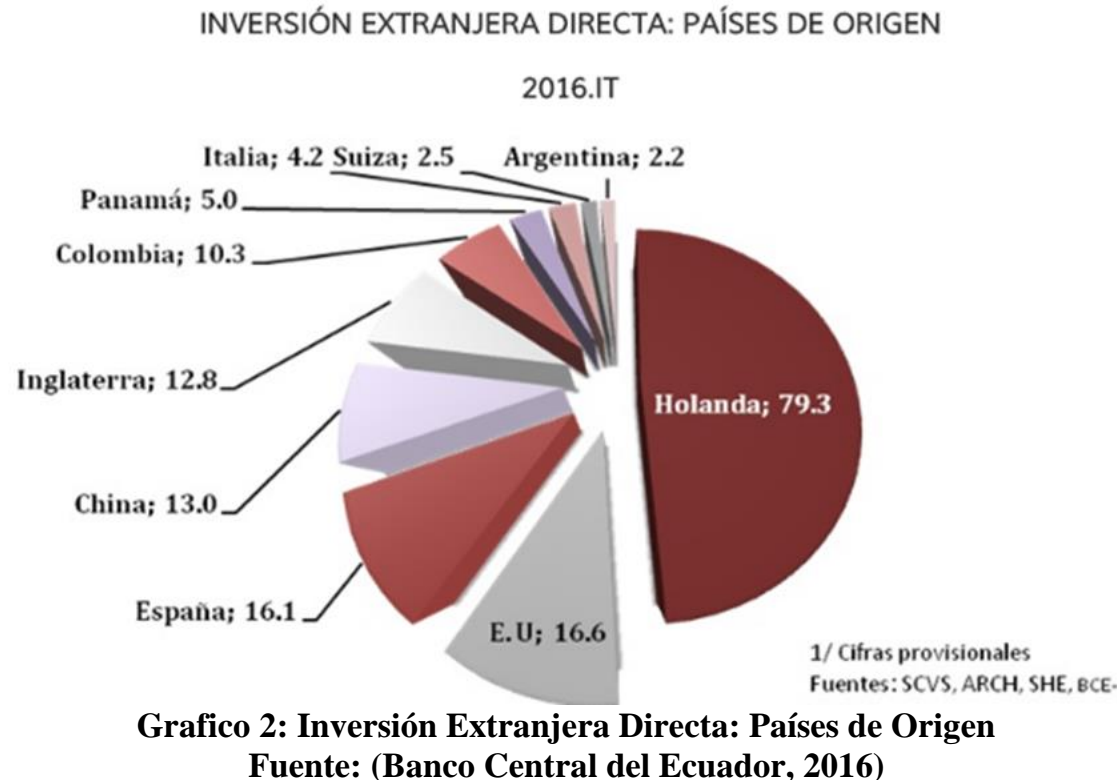


Teniendo en cuanta la relación comercial que existe entre Ecuador y Estados Unidos, siendo países en desarrollo y desarrollado respectivamente, existe la posibilidad que el país con mayor poder se sienta con derecho de mandar sobre el otro, creando relaciones comerciales inequitativas entre en los países miembros. Aunque no hay que negar que los países desarrollados por su economía y mejor administración tienen mayor poder, el rol de la OMC, es crear una atmósfera equitativa y sin discriminación alguna. Al ser la mediadora debe optar por buenas decisiones que velen por la economía del mundo manteniendo la paz en las relaciones bilaterales internacionales.

\section{Salvaguardia de tableros de fibra de madera (ECUADOR-CHILE)}

En el caso anterior, Ecuador desempeñó el papel de Reclamante, ahora analizaremos el caso de Ecuador desde la posición de Demandado para cual presentaremos "Medida de salvaguardia definitiva sobre las importaciones de tableros de fibra de madera de densidad media". La disputa empieza cuando Chile solicitó a la OMC a la celebración de consulta con Ecuador por el motivo de las importaciones de tableros de fibra de madera.

Según cifras de (Banco Central del Ecuador) las exportaciones de madera no tienen un gran peso en el comercio exterior del país pero representaron ingresos aproximadamente por 185 millones de dólares en el año 2013, siendo la madera uno de los productos más cotizados y con mejor desenvolvimiento dentro del mercado ecuatoriano. No obstante, lo que originó la aplicación de la salvaguardia fue de la empresa ecuatoriana Aglomerados Cotopaxi S.A. la cual tiene un porcentaje de $45 \%$ en la rama de producción nacional de madera (El Comercio, 2015).

Chile aseguró que no hubo amenaza de daño grave pero Ecuador no demostró el método por el cual se debería administrar y asignar las importaciones entre países proveedores, así como tampoco dio a conocer las medidas de las provisiones anteriores. Por este motivo, después de realizar dicha investigación, se impuso la medida de salvaguardia definitiva que como consecuencia inminente afectó a Chile. La OMC estableció que se hicieran consultas entre los países afectados y en el caso que no llegaran a una solución mutuamente satisfactoria en un plazo de 60 días, Chile tendría la potestad de solicitar la intervención de abogados externos para poder resolver el conflicto, o tienen otra la opción de direccionarlo a otras organizaciones para dar por terminado el caso. (OMC y Relaciones Exteriores, 2015).

Las provincias con mayor número de plantaciones de madera en general son Los Ríos, Esmeraldas, Cotopaxi, Guayas y Manabí. Por otro lado, con respecto al mercado internacional de teca , Ecuador se encuentra entre los primeros países exportadores en competencia con Colombia , Costa Rica y Panamá aunque los principales exportadores se encuentran en África y Asia (Biggs, 2003).

\section{Conclusiones y Recomendaciones}

El mundo de hoy, requiere una coordinación mucha más eficiente en el sentido macroeconómico y en la distribución de recursos más eficaces, para mejorar la producción y el manejo en sus distintas relaciones económicas y sociales. Los países siempre están en busca de mejores oportunidades y nuevas formas de negocios, es por eso que la globalización ofrece una 
interconectividad con accesos a mercados potenciales como son los de los países desarrollados. De esta manera, se diversifica el comercio mundial de una forma más dinámica y así obtener un nivel de productividad mayor en las relaciones de negocios con los demás países.

Los países no son inmunes a la globalización y a los efectos de las economías interconectadas, haciendo hincapié en el caso de Ecuador, que tiene algunas limitantes como la crisis económica y la inestabilidad jurídica para poder expandirse en el mercado extranjero, no impiden que Ecuador pueda mejorar en proyectar una buena imagen antes los demás países para que otros países deseen invertir y puedan abrir sus horizontes a nuevos retos, teniendo el antecedente de relativo éxito que es el caso del banano con Estados Unidos, teniendo como principal exportador a Estados Unidos , una potencial mundial.

La OMC en Ecuador ha desempeñado un papel altamente significativo, al mencionar anteriormente dos de los varios casos en que se ha visto involucrado y por ellos se puede, concluir que ha sido de gran aporte para el comercio ecuatoriano, incrementando el desarrollo tecnológico y la sostenibilidad en el campo económico y político.

Existen algunas críticas sobre la OMC y muchos países tienen la ideología de que esta organización contribuye al incremento de la desigualdad y pobreza a nivel mundial. La democracia es una palabra relativa dentro de un país, ya que no en todos los países existe la libre expresión y que el pueblo pueda elegir sobre sus intereses, sin embargo, al no tener derecho sobre sus decisiones los sistemas de gobierno pueden variar de un país a otro. Es por eso que la OMC tiene muchos casos de solución de diferencia provenientes de países en desarrollo, ya que los países desarrollados toman ventaja del poder que tienen e imponen aranceles y restricciones sin darse cuenta que afectan a los mercados internacionales.

Para poder garantizar que el comercio internacional sea equitativo y sostenible se necesita de cooperación de todos los países miembros. Desafortunadamente no todos los países internalizan las normas que este organismo les pone a su disposición, pero el cambio está en manos de las futuras generaciones, que deben fortalecer el sistema y la participación de la OMC en los distintos países del mundo y estar dispuestas a tomar decisiones que promuevan el buen vivir la paz y armonía en el comercio internacional.

\section{Bibliografía}

Apple. (Enero de 2012). Applesfera. Obtenido de http://www.applesfera.com/apple/disenado-encalifornia-fabricado-en-china-cambiara-eso-algun-dia-actualizado-video-explicativo

Banco Central del Ecuador. (2016). Obtenido de https://www.bce.fin.ec/index.php/boletines-deprensa-archivo/item/897-la-balanza-de-pagos-durante-el-primer-trimestre-de-2016-muestrauna-importante-reducci\%C3\%B3n-del-d\%C3\%A9ficit-comparado-con-el-trimestre-anterior

Bello, J. H. (2006). The WTO Dispute Settlement Understandings: LESS IS MORE. Obtenido de The American Journal of International Law: http://sites.fas.harvard.edu/ ec970ajf/Class_11/wto_less_is_more.pdf

Biggs, G. (2003). Solución de controversias sobre comercio e inversiones internacionales. Estados Unidos. 
Bouzas, R., \& Ffrench-Davis, R. (2000). La Globalización y la gobernalidad de los países en desarrollo. Buenos Aires.

Busch, M. L., \& Reinhardt, E. (2004). Soluciones de Diferencia en la OMC. Obtenido de http://www.oas.org/dsd/Toolkit/Documentosspa/ModuloIV/Busch\%20and\%20Reinhardt\%20Article.pdf

CEPAL. (2008). Solución de Diferencias de la OMC. En P. Gane. Santiago de Chile.

Cristian, E. (2003). El Ingreso del Ecuador a la OMC. En C. Espinosa.

DELL Company. (Agosto de 2007). DELL. Obtenido de http://es.community.dell.com/dellblogs/direct2dell/b/direct2dell/archive/2007/08/31/vista-dentro-de-la-f-brica-de-dell

Díaz, D. J., \& Regalado Eduardo. (2001). China y la OMC en la globalización. Economía y Desarrollo.

Ecuador Inmediato. (Junio de 2007). Obtenido de http://www.ecuadorinmediato.com/Noticias/news_user_view/eeuu_revoca_arancenles_antidu mpling_contra_ecuador--55164

El Comercio. (2015). Obtenido de http://www.elcomercio.com/opinion/columna-s ebastianborjasilva-organizacionmundialdelcomercio-opinion-comercio.html

Grasstek, C. V. (2013). The History and Future of the World Trade Organization. Washington.

Grossman, C. S. (2010). La Constitucionalización de la OMC.

Hoekman, B., \& Kostecki Michel. (2005). The Political Economy of the World Trading System. Oxford.

ICTSD. (Febrero de 2007). ICTSD. Obtenido de http://www.ictsd.org/bridges-news/puentes/news/loseeuu-conceden-derrota-en-conflicto-del-camar\%C3\%B3n-con-ecuador

OIC. (2016). Ministerio de Asuntos Exteriores y de Cooperación. Obtenido de http://www.exteriores.gob.es/RepresentacionesPermanentes/OficinadelasNacionesUnidas/es/ quees2/Paginas/Organismos\%20Especializados/OMC.aspx

OMC y Relaciones Exteriores. (2015). Entender la OMC. Suiza.

$\begin{array}{lllll}\text { Organización Mundial del Comercio. (2016). Obtenido de } & \end{array}$ https://www.wto.org/spanish/thewto_s/whatis_s/whatis_s.htm

Ramírez, D. (2002). Comercio Exterior: Alternativas para Ecuador. Quito.

Rose, A. K. (2003). Do we really know that the WTO increases trade? En EBSCO. American Economic Review. 